\title{
ALTERNATIVE METHOD FOR REBONDED BRACKET CLEANING USING INORGANIC SOLVENT
}

\author{
GINA MARINGKA ${ }^{1}$, BAMBANG IRAWAN ${ }^{2}$, ELLYZA HERDA $^{2 *}$, ZAINAL ALIM MASUD ${ }^{3}$
}

${ }^{1}$ Doctoral Programme, Faculty of Dentistry, Universitas Indonesia, Jakarta, Indonesia. ${ }^{2}$ Department of Dental Materials, Faculty of Dentistry, Universitas Indonesia, Jakarta, Indonesia. ${ }^{3}$ Department of Chemistry, Faculty of Mathematics and Natural Sciences, Bogor Agricultural University, Bogor, Indonesia. Email: ellyza_herda@yahoo.com

Received: 16 September 2017, Revised and Accepted: 3 October 2017

\section{ABSTRACT}

Objective: The aim of this study was to prove that organic solvents areas effective and efficient as sandblasting and burning for cleaning rebonded brackets.

Methods: Thirty stainless steel metal brackets mounted on 30 maxillary first premolar teeth were categorized into three cleaning method groups, each consisting of 10 brackets. After $24 \mathrm{~h}$, shear bond strength (SBS) tests were performed using a universal testing machine.

Results: The one-way ANOVA test showed a significant difference $(p=0.000)$ among the mean SBS values in the three cleaning method groups. However, the modified adhesive remnant index by a Kruskal-Wallis analysis showed no significant difference ( $\mathrm{p}=0.860$ ). The scanning electron microscopy results revealed that the organic solvent cleaning method showed better bracket performance than the other two methods. Moreover, the energy-dispersive X-ray spectroscopy results showed that the adhesive material remaining on the bracket base was relatively similar among the three cleaning methods.

Conclusion: Based on practicality and the results of this study, the organic solvent cleaning method was proven to be effective and efficient. Therefore, organic solvents can be recommended as an alternative cleaning method for detached brackets.

Keywords: Metal bracket, Organic solvent, Adhesive remnant index, Scanning electron microscopy, Energy dispersive X-ray spectroscopy.

(C) 2017 The Authors. Published by Innovare Academic Sciences Pvt Ltd. This is an open access article under the CC BY license (http://creativecommons. org/licenses/by/4. 0/) DOI: http://dx.doi.org/10.22159/ijap.2017.v9s2.16

\section{INTRODUCTION}

Dental bracket placement is an early and important stage of orthodontic treatment. During the orthodontic treatment period, brackets are sometimes detached from the teeth causing a disruption in the treatment. Eminkahyagil et al. stated that of every five brackets placed, one bracket will experience placement failure (or become detached [1]. Maringka and Herda conducted a survey of 500 respondents in a district health center and the Dental Hospital of the Faculty of Dentistry, Universitas Indonesia in Jakarta, that resulted in more than $90 \%$ of the respondents had experienced a detached bracket. Moreover, $60 \%$ of them occurred before the first control ( $3^{\text {rd }}$ week) after placement. They also found that one detached bracket occurred in $57 \%$ of the respondents, with 2 in $36 \%$, and 3 in $7 \%$. The detached brackets were usually located on the maxillary second premolar (60\%) [2]. In addition, the survey showed that orthodontic patients want a detached bracket to be remounted on the same day to save time [2].

According to Profit et al., bracket detachment is caused by great pressure, such as that created by chewing [3]. Wendl et al. reported that the detachment of a bracket from the tooth surface is generally caused by the failure of the bonding process between the bracket base and the adhesive material on the tooth surface [4]. It has been suggested that the factors most affecting bracket detachment are the retention form of the bracket base, the adhesive material used, and the surface of the tooth where the bracket is placed $[4,5]$.

A detached bracket needs to be cleaned before being remounted. Several techniques for cleaning detached brackets have been described, including the use of greenstone burs to clean the remaining adhesive material off the bracket base, a sandblasting method using highpressure alumina particle spray, and burning using a flame, bunsen burner, or torch [1,4-6].
The sandblasting method can increase the retention of the bracket base, but there is the possibility of a defect occurring; however, burning may cause discoloration that can make the patient uncomfortable [2]. Overall, bracket cleaning with chemicals, such as those used by OrthoCycle Co., Inc. (Hollywood, FL, USA), has not been widely used [6]. One alternative method for cleaning a detached bracket is to use an organic solvent that does not damage the bracket characteristics (physical or chemical properties), which could affect the ongoing orthodontic treatment. The polymer swelling phenomenon, preceded by solvent diffusion in the thermal process, can result in permanent damage to the polymer [7-9].

Therefore, research has been conducted to identify a solvent that can cause swelling of the adhesive material on the bracket base, so that the adhesive material becomes damaged or loose. The choice of the main solvent material was based on the composition of the adhesive material used, so this research was directed to the use of an alkaline solvent and some combination of catalysts to accelerate the adhesive material release process $[10,11]$. One organic solvent created was $\mathrm{N}$-methyl-2-pyrrolidone with a $0.1 \%$ zinc acetate catalyst that could detach the adhesive material from the bracket base $[12,13]$

The shear bond strength (SBS) is a physical property of the bracket that plays a role in orthodontic treatment, which must be clinically found in an orthodontic bracket at a magnitude of 6.8-7.9 MPa [14].

Therefore, this study was conducted to determine if organic solvent cleaning would produce a better SBS than two common cleaning methods: Sandblasting and burning. To observe the cleaning results from these three methods, scanning electron microscopy (SEM) and energy dispersive X-ray spectroscopy (EDS) were used. 


\section{METHODS}

This research study used 30 Mini Dyna Lock ${ }^{\circledR}$ stainless steel metal brackets ( 0.22 slot and $\pm 10.08 \mathrm{~mm}^{2}$ area; 3M, Monrovia, CA, USA), along with 30 maxillary first premolar teeth. This study was approved by Ethical Board from the Research Ethics Committee of the Faculty of Dentistry at the University of Indonesia (code no: 54/Ethical Approval/ FKGUI/X/2015). The tooth inclusion criteria were as follows: Cariesfree, non-fractured roots, no endodontic treatment, and extracted for orthodontic treatment reasons.

Each tooth was planted in the middle of a polyvinyl chloride cylinder (20 $\mathrm{mm}$ in height, $20 \mathrm{~mm}$ in diameter) using decorative acrylic, at a depth of $1 \mathrm{~mm}$ below the cervical line. After the acrylic hardened, dental prophylaxis was performed on the buccal surface of the tooth. The placement of the bracket began by etching the surface of the tooth with 37\% phosphoric acid (Ultra-Etch; Ultradent Products Inc., South Jordan, UT, USA) for $15 \mathrm{~s}$, rinsing with running water for $10 \mathrm{~s}$, then drying with air spray to reveal a frosty chalk-like surface. Then, the bonding was performed using adhesive material (Transbond XT Light Cure; 3M, Monrovia, CA, USA). The Transbond XT primer was smeared on the tooth enamel with a brush, while the Transbond XT paste was applied to the base of the bracket. The bracket was placed on the tooth surface parallel to the tooth axis at the height of $4 \mathrm{~mm}$ from the occlusal surface. It was pressed lightly, and any excess paste was removed with a scaler. The brackets were then cured on the mesial and distal aspects for $5 \mathrm{~s}$ each using a light-emitting diode curing unit (Elipar S10; 3M, Monrovia, CA, USA) with $850 \mathrm{~mW} / \mathrm{cm}^{2}$ irradiance. The teeth were immersed in a container with distilled water and stored for $24 \mathrm{~h}$ in an incubator (Heratherm IMH 60; Thermo Fisher Scientific, Inc., Waltham, MA, USA) set to $37^{\circ} \mathrm{C}$.

After $24 \mathrm{~h}$, an SBS test using a universal testing machine (Shimadzu AG-5000E; Kiyamachi-Nijo, Kyoto, Japan) was performed with a load of $50 \mathrm{~kg}$ and speed of $0.5 \mathrm{~mm} / \mathrm{min}$. To observe the contents of the existing elements while ensuring that the remaining adhesive material no longer remained on the surface of the bracket, both SEM and EDS (JSM6510LA; JEOL Ltd., Akishima, Tokyo, Japan) were used. The adhesive residue observations were conducted using Artun and Bergland's adhesive remnant index (ARI) modification with a score of 0-3 [15]. If all of the adhesive material was still attached to the base surface of the bracket, it was given a 3. If the adhesive material attached to the base of the bracket was more than $50 \%$, it was given a 2 . When the attached adhesive material was $\leq 50 \%$, it was given a 1 . A score of 0 showed no adhesive material attached to the surface of the bracket base. The rebonded brackets observed through SEM and EDS were scored as 3.

After the SBS test, the detached brackets were cleaned and divided into three groups ( $\mathrm{n}=10$ per group) based on the cleaning method (sandblasting, burning, and organic solvent). For this research, the sandblasting was performed using the portable sandblasting unit (Danville Materials, Carlsbad, CA, USA) with $50-\mu \mathrm{m}$ alumina particles for $15 \mathrm{~s}$ and a pressure of 5 bar. The burning method used fire in the reduction zone of a mini torch (Jet Torch- XS-0919; Shenzhen, Guangdong, China) for $5 \mathrm{~s}$ until the surface of the bracket base turned red. The organic solvent cleaning method was carried out by soaking the bracket in a closed heat-resistant container with N-methyl-2pyrrolidone and $0.1 \%$ zinc acetate as a catalyst. The container was placed in an 800 - watt microwave (MG23H3185PK, 23 L, 230 volts, $50 \mathrm{~Hz}$; Samsung, Cikarang, Jawa Barat, Indonesia) for $3 \mathrm{~min}$.

All of the cleaned brackets were put into an ultrasonic cleaner (8891; Cole-Parmer, Vernon Hills, IL, USA) for 5 min. From each group, 10 cleaned brackets (rebonded brackets) were reapplied on the same 10 teeth and attached by the same previous brackets (reused teeth). The reused teeth were cleaned using a tungsten carbide bur (Galaxy, Tampa, FL, USA) until they looked "glazed," and the bracket placement procedure was repeated again on the reused teeth.

They were stored for $24 \mathrm{~h}$ in the incubator, after which all of the brackets were repeated SBS testing and ARI observations.
The SBS values were analyzed using a one-way ANOVA and post hoc test with a significance level of $p<0.05$. The remaining adhesive material underwent a Kruskal-Wallis test with a significance level of $p<0.05$.

\section{RESULTS}

The mean and standard deviation (SD) of each of the SBS values of the control group and three cleaning method groups can be seen in Table 1.

The differences in the mean SBS values between the three cleaning methods, a post hoc Games-Howell analysis was used (Table 2), which showed a significant difference $([\mathrm{p}=0(<0.05])$. Based on the results of the post hoc test, the sandblasting method had a higher mean SBS value than the burning and organic solvent methods, but the organic solvent had a higher mean SBS value than the burning method (Table 2)

Tables 3 and 4 summarizes the observed results of the adhesive residue on the rebonded brackets after the first and second SBS tests, which used a modified ARI with the highly variable scale of $0-3$. According to the Kruskal-Wallis analysis, the modified ARI values of the three cleaning methods showed no significant differences in the residue between the methods after the first and second SBS tests.

Images of the adhesive residue on the rebonded brackets by SEM observation can be seen in Fig. 1

Fig. 2 shows images of the rebonded brackets after the sandblasting, burning, and organic solvent cleaning methods.

The EDS analysis was conducted to see the elements contained in the adhesive material that was left (score of 3) on the rebounded brackets after each of the cleaning methods. The results can be seen in Table 5 .

\section{DISCUSSION}

Based on previous research, the SBS value of a cleaned rebonded bracket should decrease by $6-20 \%$ [14]. The mean SBS value of the rebonded brackets using the sandblasting method decreased by $0.487 \mathrm{MPa}$ when compared with the mean SBS value of the control brackets. These results were similar to those of previous studies that obtained a decreased SBS value in the brackets cleaned by sandblasting; however, this difference was not significant in the previous research [16]. The study by Lunardi et al. that used the same adhesive material as this research but a different bracket type found that the SBS values were reduced after the first sandblasting, and decreased again after the seconds and blasting, showing a significant difference [17].

Similar results occurred using the burning method: When compared to the average SBS value of the control brackets, the average SBS value of the rebonded brackets was decreased by $3.519 \mathrm{MPa}$, which was a statistically significant difference $(\mathrm{p}<0.05)$. This result was similar to that of Chetan and Muralidhar research in which the SBS value in the

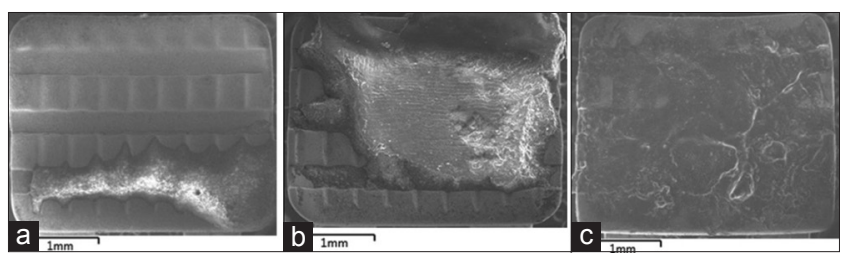

Fig. 1: $S E M$ results with $\times 30$ magnification of the adhesive residue on the rebonded brackets using the modified ARI. (a) Adhesive remnant index (ARI) score of 1 means, the attached adhesive material was $\leq \mathbf{5 0} \%$, (b) ARI score of $\mathbf{2}$ means the adhesive material attached to the base of the bracket was more than $50 \%$, and (c) all of the adhesive material was still attached to the base surface of the bracket, it was given ARI score of 3 

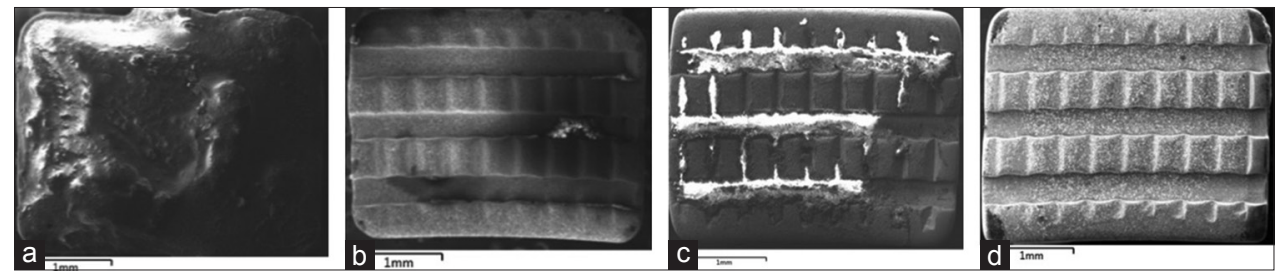

Fig. 2: Scanning electron microscopy observation results with 30x magnification after each of the cleaning methods. (a) Default rebonded bracket with full of adhesive remaining on the bracket base (ARI score of 3). The rebonded bracket in b shows a defect on the bracket base. The rebonded bracket in $\mathrm{c}$ shows adhesive remaining on the bracket base. The rebonded bracket in $\mathrm{d}$ is clean and shiny

Table 1: Descriptive statistics of the shear bond strength (MPa) of the rebonded brackets according to the three bracket cleaning method groups

\begin{tabular}{|c|c|c|c|c|c|}
\hline \multirow[t]{2}{*}{ Type of bracket } & \multirow[t]{2}{*}{$\mathbf{N}$} & \multirow[t]{2}{*}{ Mean \pm SD (MPa) } & \multicolumn{2}{|l|}{$95 \% \mathrm{CI}$} & \multirow[t]{2}{*}{$\mathbf{p}$} \\
\hline & & & Minimum & Maximum & \\
\hline New bracket, control & 30 & $15.484 \pm 0.005$ & 15.473 & 15.495 & $0.000 *$ \\
\hline Rebonded bracket, burning method & 10 & $11.965 \pm 0.072$ & 11.914 & 12.016 & \\
\hline Rebonded bracket, organic solvent method & 10 & $14.019 \pm 0.045$ & 13.987 & 14.051 & \\
\hline
\end{tabular}

*One-way ANOVA test, $\mathrm{p}<0.05$ was significant, SD: Standard deviation

Table 2: The mean SBS value differences among the sandblasting, burning, and organic solvent cleaning methods

\begin{tabular}{llll}
\hline Treatment & Treatment & Mean difference & $\mathbf{p}$ \\
\hline Sandblasting & Burning & $3.032^{*}$ & $0.000^{*}$ \\
& Solvent & $0.978^{*}$ & $0.000^{*}$ \\
Burning & Sandblasting & $-3.032^{*}$ & $0.000^{*}$ \\
& Solvent & $-2.054^{*}$ & $0.000^{*}$ \\
Solvent & Sandblasting & $-0.978^{*}$ & $0.000^{*}$ \\
& Burning & $2.054^{*}$ & $0.000^{*}$ \\
\hline
\end{tabular}

*Post hoc Games-Howell test, $\mathrm{p}<0.05$ was significant, SBS: Shear bond strength

Table 3: Frequency distribution of the ARI values of the rebonded brackets after the first SBS test

\begin{tabular}{lllllll}
\hline \multirow{2}{*}{ Rebonded bracket } & $\mathbf{n}$ & \multicolumn{7}{c}{ ARI scores } & & \\
\cline { 2 - 7 } & & $\mathbf{0}$ & $\mathbf{1}$ & $\mathbf{2}$ & $\mathbf{3}$ & $\mathbf{p}$ \\
\hline Sandblasting & 10 & 2 & 4 & 3 & 1 & 0.863 \\
Burning & 10 & 1 & 4 & 4 & 1 & \\
Organic solvent & 10 & 1 & 5 & 3 & 1 & \\
\hline
\end{tabular}

*Kruskal-Wallis test, $\mathrm{p}<0.05$ was significant, ARI: Adhesive remnant index, SBS: Shear bond strength

Table 4: Frequency distribution of the ARI values of the rebonded brackets after the second SBS test

\begin{tabular}{lllllll}
\hline Rebonded bracket & $\mathbf{n}$ & \multicolumn{7}{c}{ ARI scores } & & \\
\cline { 3 - 7 } & & $\mathbf{0}$ & $\mathbf{1}$ & $\mathbf{2}$ & $\mathbf{3}$ & $\mathbf{p}$ \\
\hline Sandblasting & 10 & 0 & 2 & 3 & 5 & 0.860 \\
Burning & 10 & 0 & 1 & 3 & 6 & \\
Organic solvent & 10 & 0 & 2 & 2 & 6 &
\end{tabular}

ARI: Adhesive remnant index, ${ }^{*}$ Kruskal-Wallis test, $p<0.05$ was significant, SBS: Shear bond strength

burning method decreased significantly [18]. According to previous studies, the burning method could cause a decrease in the SBS value of as much as $40 \%$, but in this research, the decrease was $22.7 \%$, which was statistically significant $(p=0.000)[18,19]$. The results of this research differ from those of previous studies because of the differences in the selection of the materials and the SBS measurement methods used. This research used a mini torch as a burning tool, and the SBS measurements were performed $24 \mathrm{~h}$ after the placement of the rebonded bracket on the tooth.

The rebonded brackets cleaned using the organic solvent (N-methyl2 -pyrrolidone with a $0.1 \%$ zinc acetate catalyst) showed a mean SBS value decrease of $1.465 \mathrm{MPa}$ when compared with the mean SBS value of the control brackets. Although the mean SBS value of the rebonded brackets cleaned with the organic solvent decreased, the value in this research remained far above the minimum clinical SBS requirement for an orthodontic bracket.

Based on the results of the modified ARI, the adhesive remaining on there bonded brackets after the first SBS test for each group of cleaning methods (sandblasting, burning, or organic solvent) received a score of 1 (Table 3). This suggests that the teeth exhibited good chemical retention (between the enamel and adhesive) so that the adhesive was attached mostly to enamel. The remaining adhesive based on the modified ARI after the second SBS test for each group received a score of 3 most often (Table 4). This suggests that the adhesive breakage occurred between the enamel and the adhesive material, meaning that the failure was in the adhesive [20].

This happens as a result of placing the bracket on a reused tooth, causing the chemical retention to be weak, but the mechanical retention to be strong between the bracket base and the adhesive material. Good mechanical retention occurred because of the design and shape of the base (mesh) of the bracket in such a way that the adhesive could flow over the entire mesh to create retention. The Dyna Lock ${ }^{\circledR}$ bracket used in this research has a horizontal groove as the retention form, with an open end allowing the excess adhesive flow to form adequate retention to withstand the shear force on the bracket [21].

Based on the SEM results (Fig. 2), it can be seen that with the burning method there was residual adhesive material on the bracket base, while visually the surface of the bracket base looked black. In the sandblasting method, there were visible defects on the surface of the bracket base, and the base surface was not shiny. However, with the organic solvent, the bracket base surface looked clean and shiny. This reveals that the organic solvent cleaning method provided better results than the sandblasting and burning methods.

In the EDS results (Table 5), three elements were selected to describe the adhesive material: $\mathrm{C}, \mathrm{O}$, and $\mathrm{Si}$. The increase in the $\mathrm{O}$ and $\mathrm{C}$ that remained on the bracket base after the burning method indicated that 
Table 5: EDS analysis results of the adhesive material elements (weight\%) on the rebonded brackets after the three cleaning methods

\begin{tabular}{lllll}
\hline Adhesive material elements & Rebonded bracket (Wt\%) & Methods & \\
\cline { 3 - 5 } & & Sandblasting (Wt\%) & Burning (Wt\%) & Organic solvent (Wt\%) \\
\hline C & 64.42 & 38.96 & 37.30 & 39.10 \\
O & 27.05 & 8.53 & 22.90 & 0.26 \\
Si & 8.53 & 0.15 & 0.14 & 0.26 \\
\hline
\end{tabular}

EDS: Dispersive X-ray spectroscopy

oxidation occurred during this method of cleaning. The remaining adhesive material with $\mathrm{Si}$ that ranged from $0.14 \%$ to $0.26 \mathrm{Wt} \%$ proved that all three cleaning methods were equally effective. In previous studies, it has been reported that the cleaning and enamel conditioning procedures, adhesive systems, polymerization types and times, and the moisture contamination in the enamel were the factors that could affect the retention of the metal brackets on the teeth [21-23]. Based on the SBS test results, the mean value of the rebonded brackets after the organic solvent was far above the clinical requirements of an orthodontic bracket (6.8-7.9 MPa). This value was not much different than that after the sandblasting method but was still above the mean SBS value after the burning method.

The SEM and EDS observations of the remaining adhesive showed that the organic solvent cleaning method was quite effective and efficient. In practice, when compared to the sandblasting method that requires special equipment and a special room, the organic solvent method was simpler. It only involved immersion and used a common microwave, which made this method cheaper. Moreover, when compared with the burning method, the organic solvent did not cause discoloration. Unlike the sandblasting and burning methods that must be done one bracket at a time, the organic solvent can clean many brackets at once, which improves efficiency. Overall, with regard to practicality, time, and cost, the organic solvent method can be recommended for the cleaning of detached brackets.

\section{CONCLUSION}

Based on the SBS, SEM, and EDS test results, the practicality of handling, time, and cost efficiency, and the physical appearance of the bracket after handling using $\mathrm{N}$-methyl-2-pyrrolidone with a $0.1 \%$ zinc acetate catalyst is a good alternative method for cleaning detached brackets.

\section{ACKNOWLEDGMENTS}

The authors wish to thank Dr. Ahmad Sjahriza and his team (Integrated Chemical Laboratory, Bogor Agricultural University), for their support in this research.

The publication of this manuscript is supported by Universitas Indonesia.

\section{REFERENCES}

1. Eminkahyagil N, Arman A, Cetinsahin A, Karabulut E. Effect of resinremoval methods on enamel and shear bond strength of rebonded brackets. Angle Orthod 2006;76:314-21.

2. Maringka G, Herda E. The duration of bracket detachment at public health center Jakarta and dental hospital Universitas Indonesia. J Int Dent Med Res 2016;9:345-50.

3. Profitt WR, Field HW, David MS. Contemporary Orthodontics. $4^{\text {th }}$ ed. St Louis: Mosby; 2007.

4. Wendl B, Muchitsch P, Pichelmayer M, Droschl H, Kern W. Comparative bond strength of new and reconditioned brackets and assessment of residual adhesive by light and electron microscopy. Eur J
Orthod 2011;33:288-92.

5. Yassaei S, Aghili H, Khanpayeh E, Moghadam MG. Comparison of shear bond strength of rebonded brackets with four methods of adhesive removal. Lasers Med Sci 2013;???:1-6.

6. Halwai HK, Kamble RH, Hazarey PV, Gautam V. Evaluation and comparison of the shear bond strength of rebonded orthodontic brackets with air abrasion, flaming and grinding techniques: An in vitro study. Orthodontics (Chic)2012;13:1-9.

7. Pitaressi G, Scafidi M, Alessi S, Filippo MD, Billaud C, Spadaro. Absorption kinetics and swelling stress in hydrothermally aged epoxies investigated by photoelastic image analysis. Polym Degrad Stab 2015;111:55-63.

8. Toscano A, Pitarresi G, Scafidi M, Filippo MD, Spadaro G, Alessi S. Water diffusion and swelling stresses in highly crosslinked epoxy. Polym Degrad Stab 2016;133:255-63.

9. Chang, R. Chemistry Basic: Core Concepts. Jakarta: Erlangga; 2008.

10. Man W, Xu X, Ji J, Yang Y, Shen J, Ye M, et al. The hygrothermal aging process and mechanism of the novolac epoxy resin. Composites Part B 2016;107:1-8

11. Grause G, Sugawara K, Mizoguchi T, Yoshioka T. Pyrolytic hydrolysis of polycarbonate in the presence of earth-alkali oxides and hydroxides. Polym Degrad Stab 2009;94:1119-24

12. Liu XP, Huang J, Zhang Q, Liu X, Peng H, Zhu W, et al. N-Methyl-2pyrrolidone assisted solvothermal synthesis of nano size orthorhombic lithium iron phosphate with improved Li-storage performance. J Mater Chem 2012;22:18908-14.

13. Banea MD, da Silva LF, Campilho RD. An overview of the technologies for adhesive debonding on command. Ann Dunarea De Jos Univ 2013;24:11-4

14. Quick AN, Harris AM, Joseph VP. Office reconditioning of stainless steel orthodontic attachments. Eur J Orthod 2005;27:231-6.

15. Artun J, Bergland S. Clinical trials with crystal growth conditioning as an alternative to acid-etch enamel pretreatment. Am J Orthod 1984;85:333-40.

16. Farahani M, Kabiri S, Motamedian SR, Hajighadimi M. Effect of bracket base sandblasting on bonding of orthodontic brackets on the enamel surface. Dent Hypotheses 2016;7:133-6.

17. Lunardi N, Gameiro GH, Magnani MB, Nouer DF, Siqueira VC, Consani S, et al. The effect of repeated bracket recycling on the shear bond strength of different orthodontic adhesives. Braz J Oral Sci 2008:27:1648-52.

18. Chetan GB, Muralidhar RY. Comparative Evaluation of four office reconditioning methods for orthodontic stainless steel brackets on shear bond strength-an in vitro study. Ann Essences Dent 2011;3:6-13.

19. Kachoei M, Mohammadi A, Moghaddam ME, Rikhtegaran S, Pourghaznein M, Shirazi S. Comparison of multiple rebond shear strengths of debonded brackets after preparation with sandblasting and CO2 laser. J Dent Res Dent Clin Dent Prospects 2016;10:148-54.

20. Zanarini M, Gracco A, Lattuca M, Marchionni S, Gatto MR, Bonetti GA. Bracket base remnants after orthodontic debonding. Angle Orthod 2013;83:885-91

21. Willems G, Carels CE, Verbeke G. In vitro peel/shear bond strength evaluation of orthodontic bracket base design. J Dent 1997;25:271-8.

22. Shetty V, ShekatkarY, Kumbhat N, Gautam G, Karbelkar S, Vandekar M. Bond efficacy of recycled orthodontic brackets: A comparative in vitro evaluation of two methods. Indian J Dent Res 2015;26:411-5.

23. Mehta OP, Saini S, Dahiya A. Comparative evaluation of shear bond strength of different bracket base designs in dry and wet environments. J Indian Soc Pedod Prev Dent 2008;26 Suppl 3:S104-8 